\title{
Study of High-Energy Fission in Inverse Kinematics
}

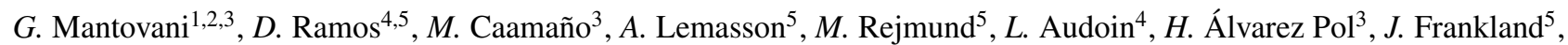
B. Fernández-Domínguez ${ }^{3}, E$. Galiana-Baldó ${ }^{3,6}, J$. Piot $^{5}, F$. Gramegna ${ }^{1}, T$. Marchi ${ }^{1}, M$. Cicerchia ${ }^{2,1}, D$. Ackermann ${ }^{5}, S$.

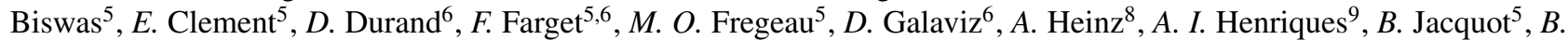

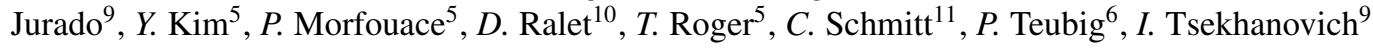

${ }^{1}$ INFN, Laboratori Nazionali di Legnaro, Legnaro (Padova), Italy.

${ }^{2}$ Universitá degli Studi di Padova, Padova, Italy.

${ }^{3}$ Universidade de Santiago de Compostela, Santiago de Compostela, Spain.

${ }^{4}$ IPN Orsay, Université de Paris-Saclay, CNRS/IN2P3, Orsay Cedex, France.

${ }^{5}$ GANIL, CEA/DRF-CNRS/IN2P3, Caen Cedex, France.

${ }^{6}$ Universidade de Lisboa, Lisboa, Portugal.

${ }^{7}$ LPC Caen, Université de Caen Basse-Normandie, ENSICAEN-CNRS/IN2P3, Caen Cedex, France.

${ }^{8}$ Chalmers University of Technology, Göteborg, Sweden.

${ }^{9}$ CENBG, IN2P3/CNRS-Université de Bordeaux, Gradignan Cedex, France.

${ }^{10} \mathrm{CSNSM}, \mathrm{CNRS} / \mathrm{IN} 2 \mathrm{P} 3$, Université de Paris-Saclay, Orsay, France.

${ }^{11}$ IPHC Strasbourg, Université de Strasbourg-CNRS/IN2P3, Strasbourg Cedex, France.

\begin{abstract}
Fission at low excitation energy, is a process in which both macroscopic and microscopic aspects are involved. Some features in the total kinetic energy and in the N/Z distributions of the fragments, commonly associated with shell effects, came out in a series of recent experiments with high excitation energy fusionfission reactions in inverse kinematics. In the latest experiment of this campaign, a study of high-energy fission and quasi-fission between a ${ }^{238} \mathrm{U}$ beam and a series of light targets was carried out by using the aforementioned technique, in order to probe the role of the shell structure in these processes.
\end{abstract}

\section{Introduction}

Since its discovery, fission appeared as a complex process where different nuclear properties interplay and shape the characteristics of the emerging fission fragment distributions [1]. Indeed, nuclear fission at low excitation energy, is a phenomenon in which both macroscopic and microscopic aspects are involved.

According to a pure liquid drop description fission should be a symmetric process. However, the large amount of experimental data on the fragment distributions reveal fission of actinides to be an asymmetric process that produces an heavy fragment with an average mass number rather constant, $\mathrm{A} \sim 140$, regardless of the identity of the fissioning system, and a light partner that complements the total mass of the system. This behaviour is understood as a consequence of structure effects on the fragments.

Therefore, fission-fragment distributions are crucial observables for modeling the fission process. Indeed, their evolution depending on the fissioning actinide and excitation energy can give hints on the role that collective and intrinsic degrees of freedom play in fission reaction dynamics. However, the access to such observables was, until recently, limited to the mass information, where the contribution of proton and neutron numbers are mixed. In fact, in experiments performed in direct kinematics, such as thermal neutron-induced fission [2-4], due to the low velocity of the fission fragments, the nuclear charge information is limited to the light fragment when is possible. So then, is not possible to ascribe the heavy-partner stabilization to a given $\mathrm{N}$ or $\mathrm{Z}$ shell-closure. Later on, it has been demonstrated that this problem can be bypassed with the use of inverse kinematics [5]. Using both mass and nuclear charge observables, measured together as isotopic fragment yields, the role of neutrons and protons in the fission process can be probed. A new experimental approach, which exploits the inverse kinematics technique to have access to the nuclear charge information and the VAMOS magnetic spectrometer in GANIL for high-resolution measurements of the fragment mass, is currently being used in a fission campaign started in 2008 [6-8]. In this approach, in-flight fission of heavy actinides, induced by transfer and fusion reactions, is used. The work presented in the following is focused on the preliminary results of the third experiment within this fission campaign.

\section{Experimental Details}

The experiment involves a ${ }^{238} \mathrm{U}^{+31}$ beam at $5.9 \mathrm{MeV} / \mathrm{u}$, with a final intensity of $10^{9} \mathrm{pps}$, impinging on a set of light targets: ${ }^{9} \mathrm{Be}\left(500 \mu \mathrm{g} / \mathrm{cm}^{2}\right) ;{ }^{\text {Natural }} \mathrm{B}\left(100 \mu \mathrm{g} / \mathrm{cm}^{2}\right) ;{ }^{24} \mathrm{Mg}$ $\left(500 \mu \mathrm{g} / \mathrm{cm}^{2}\right)$ and ${ }^{27} \mathrm{Al}\left(200 \mu \mathrm{g} / \mathrm{cm}^{2}\right)$. A series of fissioning system from ${ }^{238} \mathrm{U}$ up to ${ }^{265} \mathrm{Db}$ was populated by means of transfer and fusion reactions. 
The experimental setup, described in Fig. 1, composed by the VAMOS large-solid angle magnetic spectrometer coupled with SPIDER, a $\triangle \mathrm{E}$-E telescope which consists of two annular DSSSD and covers polar angles between $30^{\circ}$ and $47^{\circ}[10,11]$.

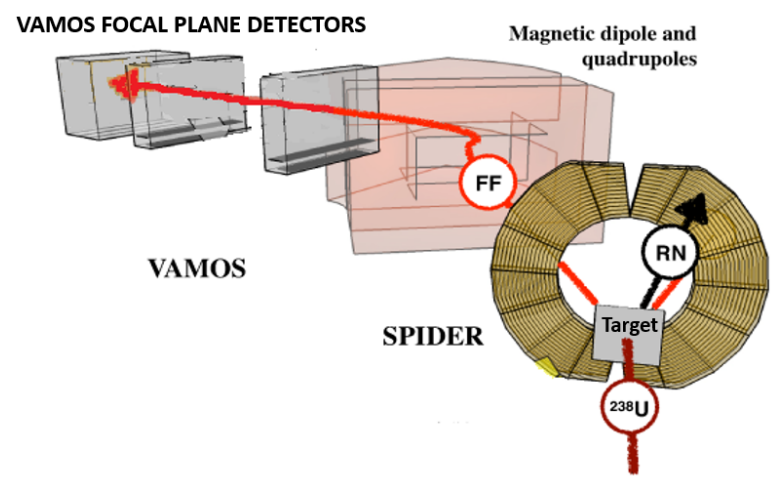

Figure 1. Sketch of the VAMOS + SPIDER experimental setup.

According to the inverse kinematics, both fission fragments were emitted at forward angles in the laboratory reference frame within a cone of $28^{\circ}$ around the beam axis which pass through the central hole of SPIDER, whereas the target-like recoil from transfer reactions was more likely emitted at higher angles and could reach the SPIDER telescope, where was isotopically identified. In such a way, the binary reaction and the excitation energy of the fissioning system were reconstructed. A fusion reaction, more frequent than any transfer channel [9], is assumed for every fission event that is registered in coincidence without any target-like recoil in the telescope. In the next section only fusion-fission event data will be discussed. When the excited compound nucleus decays by fission, one of the fragments, if the acceptance allows it, enters the VAMOS spectrometer, is deflected by the magnetic field and finally identified at the focal plane setup.

\section{Analysis and Preliminary Results}

Once the fusion-fission events have been selected using SPIDER as a veto, the identification of the fission fragments at the VAMOS focal plane setup can be performed in terms of atomic number (Z), mass number (A) and ionic charge state (q) [8]. The isotopic fission-fragment identification allows to calculate the isotopic fission-fragment yields, $\mathrm{Y}(\mathrm{Z}, \mathrm{A})$, defined as the normalized production of one particular fragment per fission. They are calculated as the number of events of each isotope $\mathrm{N}(\mathrm{Z}, \mathrm{A})$ measured during the experiment divided by the total number of produced isotopes, corrected by the geometric $\left(\epsilon_{g}^{F F}\right)$ and intrinsic $\left(\epsilon_{i}^{F F}\right)$ efficiencies for fission fragment detection.

$\epsilon_{g}^{F F}$ is determined by the angular and magnetic rigidity acceptance of VAMOS while $\epsilon_{i}^{F F}$ is the intrinsic efficiency of the detectors, both of them depending on the kinematics of the fragment.

At the VAMOS focal plane setup the detection is limited to a single fragment, and the large acceptance of VAMOS, $\pm 30 \%$ in momentum and $\pm 5.7^{\circ}$ in polar angle [10] is not sufficient to cover the entire fragment distribution.
For this reason, two different VAMOS settings were used during the experiment in order to increase the acceptance both in magnetic rigidity and in angle $(\mathrm{B} \rho=1.24 \mathrm{Tm}, \theta=$ $14^{\circ} ; \mathrm{B} \rho=1.1 \mathrm{Tm}, \theta=21.5^{\circ}$ ). This is translated in a wider covered range of fragment masses.

The geometric efficiency is given by the product of the acceptance in polar angle $\mathrm{f}_{\theta_{F F}^{C M}}\left(A, \theta_{F S}, \beta_{F S}\right)$, for the acceptance in azimuthal angle, $\mathrm{f}_{\phi}\left(\theta_{F F}^{l a b}, \delta\right)$. The latter must be calculated for each $\left(\theta_{F F}^{l a b}, \delta\right)$ selected window as the ratio between the range of $\phi_{l a b}$ accepted in VAMOS and the total physical $\phi_{\text {lab }}$ range covered by fission fragments:

$$
\frac{f_{\phi}\left(\theta_{F F}^{l a b}, \delta\right)=\left|\phi_{\max }^{l a b}-\phi_{\min }^{l a b}\right|}{2 \pi}
$$

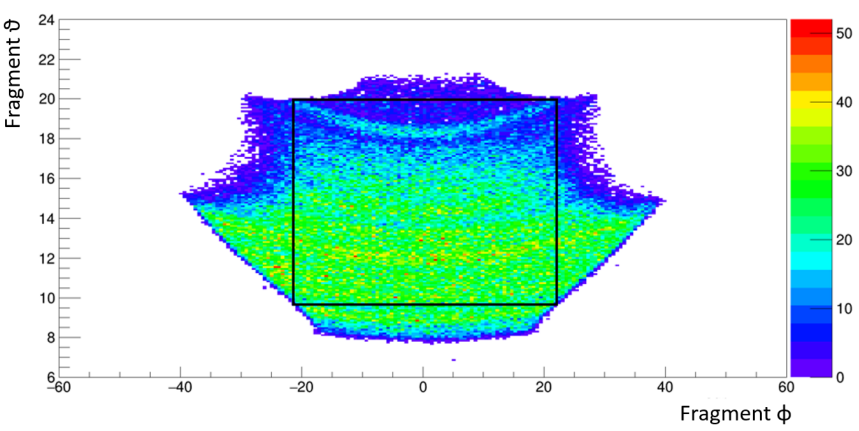

Figure 2. Selected window in $\theta$ and $\phi$ for a central $\mathrm{B} \rho$ range ( $\delta$ $=0.9975-1.0025)$ for VAMOS setting $\mathrm{B} \rho=1.24 \mathrm{Tm}, \theta=14^{\circ}$ to calculate the $\phi$-angular acceptance, $\mathrm{f}_{\phi}\left(\theta_{F F}^{l a b}, \delta\right)$.

In Fig. 2 is reported an example of a selected window in $\left(\theta_{F F}^{l a b}, \delta\right)$ for the $\mathrm{f}_{\phi}$ acceptance calculation.

\section{Conclusions and Perspectives}

The inverse kinematics technique allows to identify the fission fragments both in mass and nuclear charge. $\phi$ - and $\theta$ angular acceptance calculations, needed to obtain the fission fragment yields, are still in progress. A further step is necessary to characterize the scission point in terms of fission fragment velocities and masses, in order to calculate the total kinetic energy and the total excitation energy.

\section{References}

[1] N. Bohr and J. A. Wheeler, Phys. Rev. 56, 426 (1939)

[2] W. Lang et al., Nucl. Phys. A 345, 34 (1980)

[3] U. Quade et al., Nucl. Phys. A 487, 1 (1988)

[4] J. P. Bocquet et al., Z. Phys. A 335, 41 (1990)

[5] K.-H. Schmidt et al., Nucl. Phys. A 665, 221 (2000)

[6] M. Caamaño et al., Phys. Rev. C 88, 024605 (2013)

[7] M. Caamaño et al., Phys. Rev. C 92, 034606 (2015)

[8] D. Ramos et al., Phys. Rev. C 97, 054612 (2018)

[9] D. C. Biswas et al., Phys. Rev. C 56, 1926 (1997)

[10] M. Rejmund et al., Nucl. Instr. and Meth. in Physics Research Section A 646, 184 (2011)

[11] M. Vandebrouck et al., Nucl. Instr. and Meth. in Physics Research Section A 812, 112 (2016) 